\title{
Magnetic hysteresis in the Cu-Al-Mn intermetallic alloy: Experiments and modeling
}

\author{
Eduard Obradó, Eduard Vives, and Antoni Planes \\ Departament d'Estructura i Constiuents de la Matèria, Facultat de Física, Universitat de Barcelona, Diagonal 647, \\ 08028 Barcelona, Catalonia, Spain
}

(Received 9 November 1998)

\begin{abstract}
We study isothermal magnetization processes in the $\mathrm{Cu}-\mathrm{Al}-\mathrm{Mn}$ intermetallic alloy. Hysteresis is observed at temperatures below the spin-freezing of the system. The characteristics of the hysteresis cycles as a function of temperature and $\mathrm{Mn}$ content (magnetic element) are obtained. At low temperature $(\$ 5 \mathrm{~K})$ a change from smooth to sharp cycles is observed with increasing Mn content, which is related to the decrease of configurational disorder. We also study a zero-temperature site-diluted Ising model, suitable for the description of this $\mathrm{Cu}-\mathrm{Al}-\mathrm{Mn}$ system. The model reproduces the main features of the hysteresis loops observed experimentally. It exhibits a disorder-induced critical line separating a disordered phase from an incipient ferromagnetic ground state. The comparison between the model and the experiments allows us to conclude that the observed change in the experimental hysteresis loops can be understood within the framework of the theory of disorder-induced criticality in fluctuationless first-order phase transitions. [S0163-1829(99)13821-9]
\end{abstract}

\section{INTRODUCTION}

Hysteresis is the history dependence of the properties of a material when driven by an applied external field. ${ }^{1}$ It is observed in many different systems in nonequilibrium conditions. In general it is a dynamical effect which must be treated within a time-dependent formalism. Here we will focus on a special class of systems that display, under certain conditions, time-independent hysteresis properties. The prototypes are ferromagnets, at low temperature, in which magnetization versus magnetic field paths are not influenced by the applied field rate, at least for low enough rates. In such materials, when these paths are studied in detail, it is commonly observed that they are not continuous but rather composed of small bursts or avalanches: this is the so-called Barkhausen noise. ${ }^{2}$ This abrupt and stochastic response of the magnetization to a continuously varying external field has been related to the disorder existing in the system., Actually, domain walls are pinned by impurities and therefore a magnetization change can only occur if the corresponding energetic barriers are overcome (depinning). Since these barriers are large, thermal activation is not effective, and the evolution of the sytem can only proceed by modifying the external field (athermal behavior). In addition to ferromagnetic materials, a wide variety of complex systems such as martensitic materials, ${ }^{5}$ superconducting films, ${ }^{6}$ or capillary condensation systems, ${ }^{7}$ display similar phenomenology. All these systems can be described in the framework of fluctuationless first-order (FLFO) phase transitions, i.e., they exhibit, when driven by an external field, a firstorder phase transition influenced by disorder with irrelevant thermal fluctuations.

Recently, different versions of spin lattice models with disorder, as the random bond ${ }^{8-10}$ and random field Ising models, ${ }^{11}$ have been used to model hysteresis and avalanches in systems undergoing FLFO phase transitions. Deterministic algorithms with synchronous dynamics corresponding to local energy relaxation have been employed to study hysteresis paths at zero temperature. It has been found that the shape of hysteresis loops depends on the amount of disorder. ${ }^{9,11}$ For low disorder, when sweeping the field from $-\infty$ to $+\infty$, the system reverses sharply the magnetization (infinite avalanche) at a given field, while for a high amount of disorder the path is smoother and extends over a wide range of fields. Interestingly the change in the hysteresis cycle shape has been attributed to the existence of a critical point at a given degree of disorder, where the infinite avalanche disappears. For such a degree of disorder, avalanches of all sizes are detected indicating that the system behaves critically: the avalanche size distribution follows a power law characterized by a universal exponent depending only on the space dimensionality. ${ }^{10}$ Actually, in agreement with the models, it has been experimentally observed that the amplitude of Barkhausen pulses in ferromagnetic materials, and the amplitude of the acoustic signals generated during martensitic transitions, extend over several decades indicating the possibility of a universal critical behavior. ${ }^{4,5,12,13}$ A number of attempts to make a comparison between models and experiments have been reported. ${ }^{14,15}$ Nevertheless, the existence of criticality induced by changing the amount of disorder has not been confirmed. The difficulty arises from the possibility of controlling the amount of disorder in a given system. In any case, it must be mentioned that the studied models appear as very simple idealizations when compared to the complexity inherent to real systems.

This paper is concerned with the study of the magnetic properties of the $\mathrm{Cu}-\mathrm{Al}-\mathrm{Mn}$ intermetallic alloy. After a suitable heat treatment, ${ }^{16}$ this material displays an $L 2_{1}$ structure. The stoichiometric compound $\mathrm{Cu}_{2} \mathrm{AlMn}$ is ferromagnetic with a Curie temperature of $630 \mathrm{~K}$ and it is known that the entire magnetic moment of the system must be attributed to the $\mathrm{Mn}$ atoms ${ }^{17}$ coupled via the Ruderman-Kittel-KasuyaYosida (RKKY) interaction. ${ }^{18}$ ac susceptibility measurements have revealed the existence of a spin-freezing process at low temperatures. This freezing has been shown to correspond to a spin glass phase transition for low enough Mn content. ${ }^{19}$ Beyond this problem, here we focus on the study of the low-temperature magnetic hysteresis for such systems. 


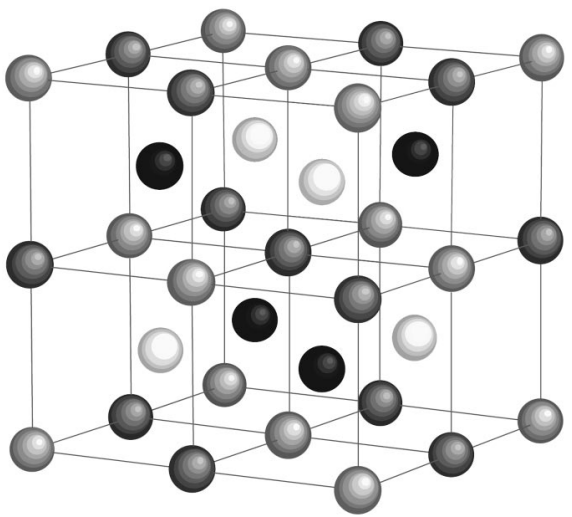

FIG. 1. Crystal structure of $\mathrm{Cu}_{2} \mathrm{AlMn}$. Black spheres: $\alpha(\mathrm{Mn})$ sublattice. White spheres: $\beta(\mathrm{Al})$ sublattice. Grey spheres: $\gamma$ and $\delta$ $(\mathrm{Cu})$ sublattices.

We have studied a family of composition related alloys which undergo, prior to the spin-freezing process, a diffusionless structural transition to a close-packed phase (martensitic transition). In these materials, magnetic disorder has two main origins: the lack of stoichiometry and the existence of incomplete configurational ordering. ${ }^{20,21}$ The important fact is that the Mn configurational disorder increases as the $\mathrm{Mn}$ content decreases. We show that increasing the degree of disorder, the low-temperature hysteresis cycle changes from sharp to smooth. This change is suggested to be related to a disorder induced phase transition. Since the experimental evidences are not definitively conclusive, we reinforce our interpretation by developing a microscopic model for such site-diluted magnetic systems. By fitting the model parameters to $\mathrm{Cu}-\mathrm{Al}-\mathrm{Mn}$ available data, we reproduce approximately the observed phenomenon and associate it with the disorder induced phase transition. The paper is organized as follows. In Sec. II, we present the experimental results and in Sec. III we introduce a model based on a site diluted Ising model suitable for the study of hysteresis in spin glass systems and compare the results obtained numerically with the experimental ones corresponding to $\mathrm{Cu}-\mathrm{Al}-\mathrm{Mn}$. Finally, in Sec. IV we discuss the results and conclude.

\section{EXPERIMENTAL RESULTS}

We have studied a family of alloys, with compositions close to the $\mathrm{Cu}_{3-x} \mathrm{AlMn}_{x}$ line. Polycrystalline (grain size $\sim 100 \mu \mathrm{m}$ ) cylindrical-shaped ingots were obtained by melting pure elements $(99.99 \%)$. Rectangular shaped samples $\left(9 \times 3.5 \mathrm{~mm}^{2}\right)$ were cut using a low-speed diamond saw. The samples were mechanically polished down to a thickness of $0.1 \mathrm{~mm}$. Samples were annealed for $900 \mathrm{~s}$ at $1073 \mathrm{~K}$, quenched into a mixture of ice and water, and annealed at room temperature for several weeks. This heat treatment leads to a long-lived metastable state in which the material displays an $L 2{ }_{1}$ structure ( $F m 3 m$ space group). This structure can be viewed as defined on a bcc lattice divided in four distinguishable fcc sublattices ( $\alpha, \beta, \gamma$, and $\delta$ ) as shown in Fig. 1. For samples with $x<1$ (as in the present work), sublattices $\gamma$ and $\delta$ (for instance) are filled with $\mathrm{Cu}$ atoms, and $\mathrm{Mn}$ atoms sit preferentially in sublattice $\alpha$. However, for kinetic reasons, a fraction of $\mathrm{Mn}$ atoms also occupies the $\beta$
TABLE I. Composition, characteristic spin-freezing temperatures, paramagnetic Curie temperatures, and Martensitic transition temperatures of the samples investigated.

\begin{tabular}{lccc}
\hline \hline Alloy composition & $T_{f}(\mathrm{~K})$ & $\theta_{c}(\mathrm{~K})$ & $T_{M}(\mathrm{~K})$ \\
\hline $\mathrm{Cu}_{2.884} \mathrm{Al}_{0.996} \mathrm{Mn}_{0.120}$ & $15 \pm 1$ & $101 \pm 10$ & 390 \\
$\mathrm{Cu}_{2.804} \mathrm{Al}_{0.944} \mathrm{Mn}_{0.252}$ & $31 \pm 1$ & $138 \pm 10$ & 251 \\
$\mathrm{Cu}_{2.728} \mathrm{Al}_{0.912} \mathrm{Mn}_{0.360}$ & $45 \pm 1$ & $159 \pm 10$ & 133 \\
\hline \hline
\end{tabular}

sublattice. Experiments based on atom location by channelling enhanced microanalysis (ALCHEMI) performed on similar samples have shown that almost no Mn atoms occupy sublattices $\gamma$ and $\delta .^{20}$

The spin-freezing temperatures $T_{f}$ of the alloys studied have been obtained from ac-susceptibility measurements. ${ }^{19}$ As shown in Table I, in all the samples studied $T_{f}$ is below the martensitic transition. We also present the values of the paramagnetic Curie temperature $\theta_{c}$ and the temperature $T_{M}$ of the structural transition taken from the same Ref. 19. Concerning $\theta_{c}$, note that it is positive for the three samples, which indicates the mean ferromagnetic character of the interactions.

Magnetization measurements have been carried out, at low field rates $(<10 \mathrm{Oe} / \mathrm{s})$ using a superconducting quantum interference device magnetometer enabling applied fields up to $5 \mathrm{~T}$ (the relative error in the field control is $\pm 0.1 \%)$. The hysteresis observed in the glassy phase reflects the nonequilibrium state of the system characterized by a logarithmically slow response of the magnetization to an applied field. Limiting hysteresis loops have been measured after a zero-field cooling (ZFC) and subsequent (isothermal) field sweep between -5 and $+5 \mathrm{~T}$ at different temperatures (controlled within $\pm 0.01 \mathrm{~K}$ ). In Fig. 2 we show the hysteresis cycles obtained at $5 \mathrm{~K}$ in the three samples studied. Loops are symmetrical and display small coercivity and remanence. It is worth mentioning that for the sample with $x$ $=0.25(6.3 \% \mathrm{Mn})$, the hysteresis loop has also been measured after a field cooling (FC). The cycle obtained is identical from the one measured after a ZFC. This proves the absence of global uniaxial exchange anisotropy in the polycrystalline samples considered.

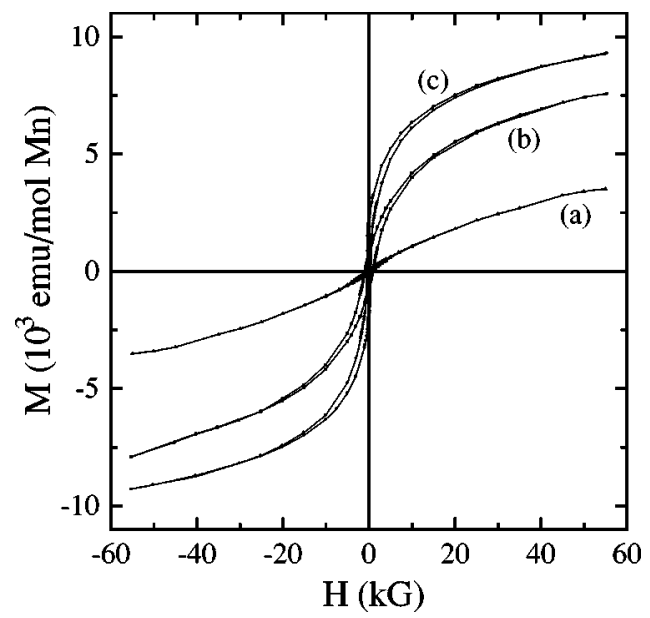

FIG. 2. Hysteresis cycles at $T=5 \mathrm{~K}$ at the three different compositions studied: $x=0.12$ (a), $x=0.25$ (b), and $x=0.36$ (c). 


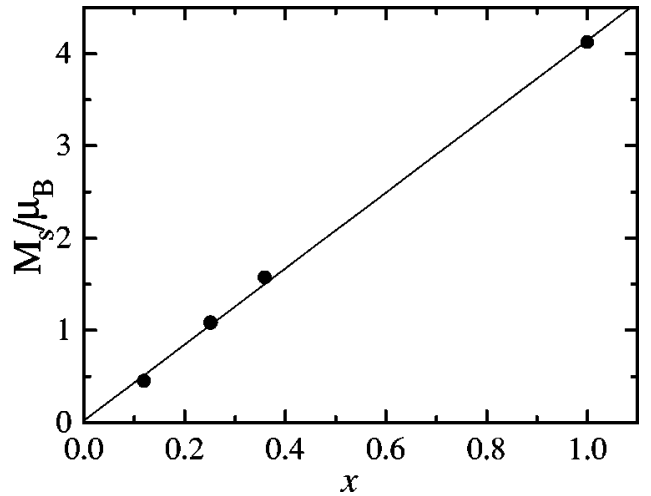

FIG. 3. Saturation magnetization as a function of $x$. The continuous line shows a linear fit.

From the results given in Fig. 2 it is clear that the magnetization can be supposed to be composed of irreversible and reversible contributions ${ }^{22}$

$$
M=M_{\text {rev }}+M_{\text {irr }} .
$$

Only the irreversible term contributes to hysteresis. Actually, the reversible contribution must be ascribed to the competition of the external field with minoritary antiferromagnetic bonds in the system, and can be assumed to be linear with the magnetic field

$$
M_{\mathrm{rev}}=\chi(T) H .
$$

From linear fitting, we have determined $\chi$ for the alloys investigated. The values obtained are $\chi=0.029 \pm 0.010 \mathrm{emu} /$ $(\mathrm{mol} \mathrm{Mn} \mathrm{Oe}), \chi=0.041 \pm 0.010 \mathrm{emu} /(\mathrm{mol} \mathrm{Mn} \mathrm{Oe})$, and $\chi$ $=0.023 \pm 0.010 \mathrm{emu} /(\mathrm{mol} \mathrm{Mn} \mathrm{Oe})$ for the samples with $x$ $=0.12(3 \% \mathrm{Mn}), x=0.25(6.3 \% \mathrm{Mn})$, and $x=0.36(9 \%$ $\mathrm{Mn})$, respectively. Therefore, within errors, $\chi$ can be assumed independent of $x$, at least in the range of compositions studied. After subtraction of the reversible term in the magnetization measured, we have estimated a saturation magnetization $M_{s}$. It is represented (per Mn atom) as a function of $x$ in Fig. 3. We obtain that $M_{s}$ increases linearly with $x$. The value of $M_{s}$ corresponding to the stoichiometric $\mathrm{Cu}_{2} \mathrm{AlMn}$ alloy $\left[M_{s}(x=1) \equiv \mu \simeq 4.1 \mu_{B}\right]$ has been taken from the literature. ${ }^{S}$

As temperature is increased the width of the cycle (coercivity) decreases and, as expected, we have not observed hysteresis above $T_{g}$. In Fig. 4 we show the effect of temperature on hysteresis by plotting the central part of the hysteresis loops at three different temperatures. In all cases the cycles are smooth and no discontinuities (or avalanches) have been detected. However, the cycle corresponding to $x$ $=0.36$ obtained at $T=5 \mathrm{~K}$, shows practically a complete reversal of the remanent magnetization in a very reduced interval of fields. This changes the shape of the hysteresis loop which becomes sharper in its central part.

In order to quantitatively characterize the shape of the hysteresis loops, we have represented in Fig. 5 the remanence $M_{r}$, the coercivity $H_{c}$ and the area of the loops $E$, as a function of temperature for the three different compositions studied. The area of the loops, which gives the dissipated energy per cycle, decreases with $T$ and increases with the Mn content $x$. Notice also that for the sample with $x=0.36$ the

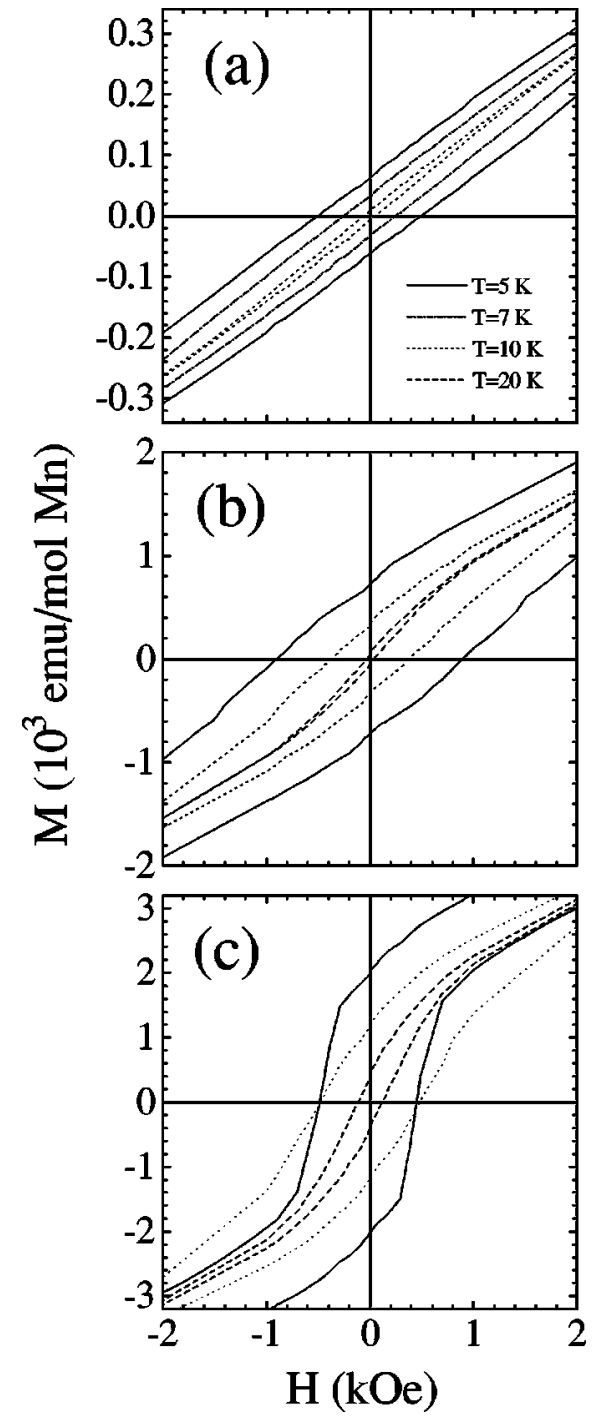

FIG. 4. Central part of the hysteresis cycles for three diferent temperatures and compositions: $x=0.12$ (a), $x=0.25$ (b), and $x$ $=0.36(\mathrm{c})$.

coercive field does not change for $T<10 \mathrm{~K}$. This fact is associated with the change in the shape of the hysteresis loop outlined in the previous paragraph.

We argue that the observed change in the hysteresis loop can be related to the fact that, as has recently been shown, ${ }^{19}$ a spin-glass phase transition occurs for samples with $x$ $<0.3$, but not for samples with higher Mn concentration. For these compositions, the cusp in the magnetic susceptibility curve as a function of temperature is related to the freezing of ferromagnetic clusters, being the high-temperature phase superparamagnetic and the low-temperature phase what we will call in this article a ferromagnetic spin-glass. ${ }^{23}$ Therefore we suggest that the change in the hysteresis loop may be related to a phase transition which has the origin on the different ground state properties of the system as $x$ is increased. Actually the value of $x$ at the transition point, should be close to the percolation limit for the system studied. It would be expected that remanent magnetization reverses at a single value of the field (large avalanche). The fact that this does not strictly occur is due, as we will discuss in the next section, to the polycrystalline character of the samples studied. 


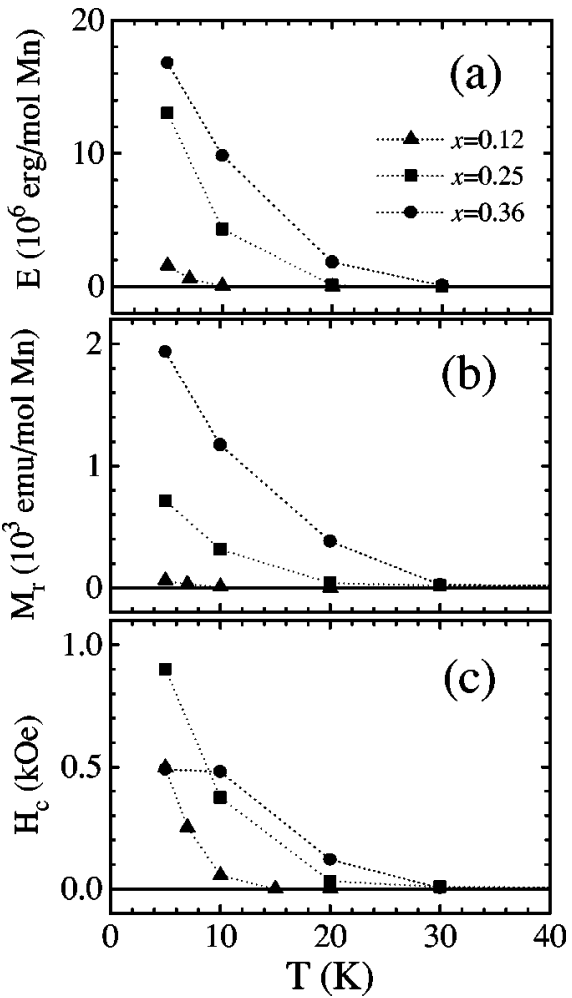

FIG. 5. Hysteresis cycle properties versus temperature for three different compositions: dissipated energy (a), remanent magnetization (b), and coercive field (c).

We have also studied first-order reversal cycles within the limiting loop obtained as follows: starting from $H=-5 \mathrm{~T}$ the external field is increased to a value $H_{\max }\left(H_{c}<H_{\max }\right.$ $<5 \mathrm{~T}$ ), before saturation. At this point the field evolution is reversed back to $H=-5 \mathrm{~T}$. For different $H_{\max }$ one obtains a family of trajectories, some of which are displayed in Fig. 6

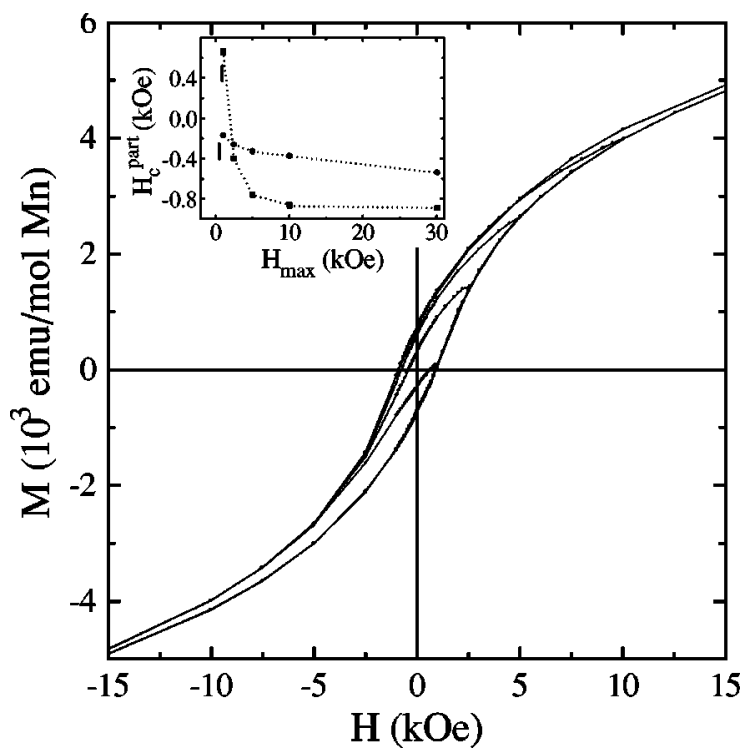

FIG. 6. Partial loops corresponding to a sample with $x=0.25$ and $T=5 \mathrm{~K}$. The inset shows the behavior of the coercive field $H_{c}^{\text {part }}$ as a function of $H_{\max }$ for the samples with $x=0.25$ (squares) and $x=0.36$ (circles). The vertical bars show the values of the coercive field for the limiting loops in each case.

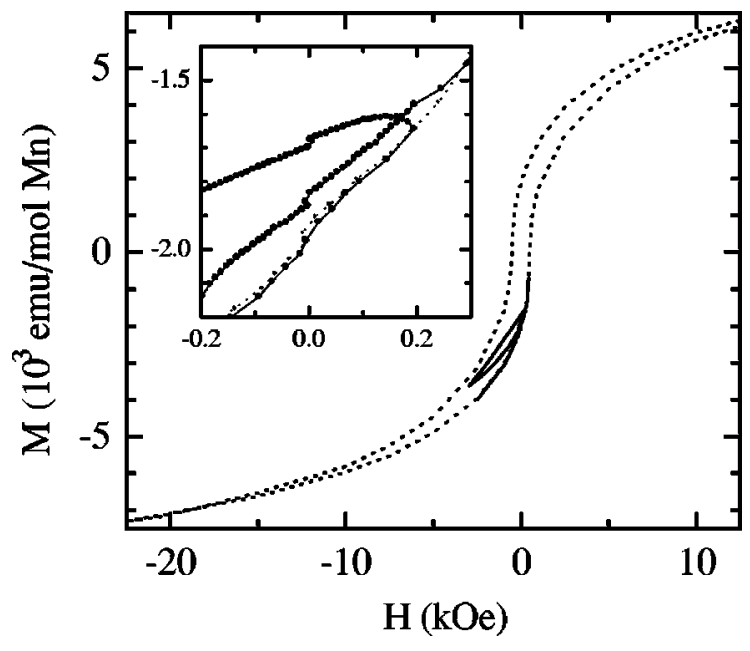

FIG. 7. Internal loop corresponding to a sample with $x=0.36$ and $T=5 \mathrm{~K}$. The inset shows a detail of the turning point.

for the sample with $x=0.25$ and $T=5 \mathrm{~K}$. It is worth noting that in all cases the decreasing reversal trajectories join tangentially the limiting cycle. The shape of these reversal trajectories depends on $H_{\max }$. In the inset of Fig. 6 we show the coercive field of the reversal trajectories $H_{c}^{\text {part }}$ as a function of $H_{\max }$. Note that for the sample with $x=0.36$ (circles), $H_{c}^{\text {part }}$ remains approximately constant with $H_{\max }$ when aproaching $H_{c}$ from above. This is indicative of the fact that the reversal of magnetization occurs at a fixed field for high $x$.

An interesting feature observed in a number of systems exhibiting FLFO phase transitions is the return point memory effect. This property is well illustrated by analyzing the characteristics of complete internal hysteresis loops. These internal loops are obtained when, coming from $H$ $=-5 \mathrm{~T}$ and being in a state corresponding to a field $H_{1}$ $<5 \mathrm{~T}$ on the limiting cycle, the field evolution is reversed back until reaching $H_{2}<H_{1}$, and later is increased up again to $H_{1}$. The return point memory effect is verified if, after this path, the system returns exactly to the same state that was left in the limiting cycle, that is, if it has memory of the former state. In this case, the same memory effect extends to any subcycle within a given cycle, and therefore this indicates that the state of the system can remember an entire hierarchy of turning points in its past evolution. This phenomenon has been observed in some, but by no means all, systems with FLFO phase transitions.

We have performed such complete internal cycles by reversing the field at different states in the limiting cycle. Typical results are shown in Fig. 7. It is observed that after performing an internal loop and returning to the former field, the magnetization has increased. By performing internal cycles at different sweeping field rates (in the positive and negative applied field regions), we have checked that this increase of the magnetization is significantly larger than the increase expected from logarithmic relaxational effects. This enables us to conclude that, in our system, the return point memory property is not satisfied.

\section{MODELING}

In order to gain understanding of the observed experimental phenomena we develop, in this section, a model contain- 
TABLE II. Exchange constants for the different neighbors on the simple cubic lattice formed by sublattices $\alpha$ and $\beta$. The values with asterisk have been interpolated as explained in the text, while the others have been taken from Ref. 18.

\begin{tabular}{lccc}
\hline \hline Neighbor & Position & Distance $(a)$ & $J_{i j}(\mathrm{meV})$ \\
\hline 1 & $(100)$ & 1 & $-2.098^{*}$ \\
2 & $(110)$ & $\sqrt{2}$ & 0.744 \\
3 & $(111)$ & $\sqrt{3}$ & $0.399^{*}$ \\
4 & $(200)$ & 2 & 0.296 \\
5 & $(210)$ & $\sqrt{5}$ & $-0.2343^{*}$ \\
6 & $(211)$ & $\sqrt{6}$ & 0.355 \\
7 & $(220)$ & $\sqrt{8}$ & -0.411 \\
\hline \hline
\end{tabular}

ing two basic ingredients: randomness of the lattice position of the magnetic atoms and competing interactions. These two ingredients are crucial for the existence of spin-glass phenomena. $^{24}$ The proposed model is a modified version of the diluted Ising model at $T=0 .^{25}$

We only consider sublattices $\alpha$ and $\beta$ as possible sites for Mn atoms (see Fig. 1). Therefore we define a simple cubic lattice $^{26}$ with $i=1, \ldots, N\left(N=L^{3}\right)$ sites and with periodic boundary conditions and lattice spacing $a$. On each site we define a variable $c_{i}=1,0$ which indicates the presence (1) or absence (0) of $\mathrm{Mn}$ on that site, and an Ising variable $S_{i}= \pm 1$ which accounts for two possible magnetic states of $\mathrm{Mn}$ atoms. The Hamiltonian of the model is written as

$$
\mathcal{H}=\sum_{i j} J_{i j} c_{i} c_{j} S_{i} S_{j}-\mu H \sum_{i} c_{i} S_{i},
$$

where the first term is a sum over site pairs, $J_{i j}$ is the exchange energy, $H$ the external field, and $\mu$ the effective magnetic moment per atom. A number $N_{\mathrm{Mn}}=\Sigma_{i} c_{i}$ of Mn atoms are randomly placed as follows: a fraction $(1-p) N_{\mathrm{Mn}}$ is placed in sublattice $\alpha$ while the rest $p N_{\mathrm{Mn}}$ is "misplaced" in sublattice $\beta$. Values of $p$ ranging from 0 to 0.5 have been considered. The concentration of $\mathrm{Mn}$ is given by $x$ $=N_{\mathrm{Mn}} /(2 N)$. The exchange constants $J_{i j}$ are distance dependent. For the stoichiometric alloy, $J_{i j}$ have been fitted from inelastic neutron scattering experiments. ${ }^{18}$ The fitted values are the ones corresponding to neighbors of second, fourth, sixth, and seventh order in Table II. We assume that $J_{i j}$ do not change significantly when altering the Mn content. Nevertheless we still have to interpolate the values of the $J_{i j}$ corresponding to distances which are not present in perfectly ordered stoichiometric alloys. To do that we have fitted a standard RKKY dependence to the experimental $J_{i j}$ :

$$
J(r)=A \frac{\cos [B(r / a)]}{(r / a)^{3}},
$$

where $B$ is proportional to the Fermi moment $B=2 k_{F} a$. Since it is difficult to fit such an oscillating function we have first checked different values of $B$ in order to get the correct signs of the $J_{i j}$ found experimentally. The signs are only correctly fitted for values of $B$ around $\sim 19.64 .^{27}$ A standard least square fitting, then, renders $A=-2.58 \mathrm{meV}$. Using this fit we then interpolate the values of $J_{i j}$ for the neighbors of first, third, and fifth order indicated in Table II with asterisks.
A strong antiferromagnetic interaction between n.n. sites (that can only occur due to misplaced Mn atoms) is obtained. This is consistent with the antiferromagnetic character found in $\gamma$-Mn with fcc structure ${ }^{28}$ (lattice parameter $<2 a$ ). Nevertheless we have checked that the mean character of the interactions (which can be estimated as a sum $\sum n_{i j} J_{i j}$ over all Mn pairs $n_{i j}$ ) is always ferromagnetic for all values of $x$ and $p$. This is consistent with the positive paramagnetic $\mathrm{Cu}$ rie temperatures obtained experimentally (see Table I).

For the simulations we consider the exchange energy for next-nearest neighbors $J_{2}=0.744 \mathrm{meV}$ as the energy unit. We define a reduced Hamiltonian as

$$
\mathcal{H}^{*}=\sum_{i j} J_{i j}^{*} c_{i} c_{j} S_{i} S_{j}+H^{*} \sum_{i} c_{i} S_{i}
$$

where $J_{i j}^{*}=J_{i j} / J_{2}$ and $H^{*}=\mu H / J_{2}$. We define the reduced magnetization as

$$
m^{*}=\frac{\sum_{i} S_{i}}{N / 2} .
$$

With this definition, the maximum reduced magnetization for the stoichiometric alloy is $m^{*}=1$.

Hysteresis cycles $\left[m^{*}\left(H^{*}\right)\right]$ are obtained by starting with all the spin variables $S_{i}=-1$ and the external field $H^{*}$ $=-\infty$. The field is then slowly increased until a spin becomes locally unstable, i.e., there is a decrease of energy if it is flipped. Then the external field is stopped, the spin is flipped and an avalanche starts: more spins may become unstable. These are simulatenously flipped, which may trigger some new unstable spins, and so on until the avalanche stops. The number of steps necessary to reach this stable situation defines the avalanche duration and the corresponding magnetization change the avalanche size. Then the external field is increased again. This is the so-called synchronous dynamics. $^{29}$

\section{A. Results}

We have studied lattices with $L=32$ and for many runs, averages over 50 different initial random atomic configurations have been taken. We have systematically studied systems with concentration $x=0.125,0.25,0.375$, and 0.5 and disorder values $p=0.1,0.2,0.3,0.4$. Examples of the hysteresis cycles are shown in Fig. 8. For a fixed value of $p$, the hysteresis loop becomes sharper as $x$ is increased, while for a fixed value of $x$ the loop becomes smoother when increasing $p$. Note also that for high values of $p$ and $x$ the loops become antiferromagneticlike with long tails and with a tendency to split into two subloops. In all cases, when looking in detail at the loops, they consist of a sequence of discrete jumps (avalanches) that join metastable states. This is illustrated in Fig. 9. Due to the existence of antiferromagnetic interactions, inverse avalanches, decreasing the magnetization when increasing the field, may occur. Concerning this point, one should remember that the evolution is controlled by a local (and not global) equilibrium condition.

In order to study in more detail the evolution from smooth to sharp hysteresis cycles we have studied the statistical distribution of avalanche sizes. Sharp loops will exhibit few 


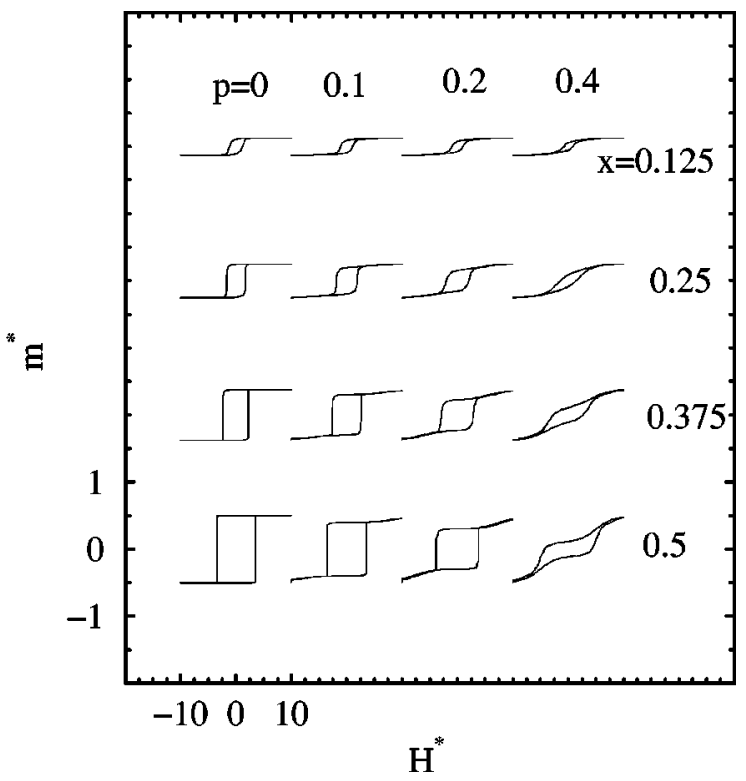

FIG. 8. Hysteresis cycles for different values of $p$ and $x$ as indicated.

large avalanches while smooth loops will exhibit small avalanches only. The avalanche distributions have been computed by averaging over 50 different realizations of the random distribution of $\mathrm{Mn}$ atoms. The resulting histograms are presented in Fig. 10 for different values of $p$ and $x$. We find that, in this $p$ - $x$ diagram, a critical line exists at which avalanches of all sizes are observed and the distributions are power law. This behavior has been analyzed following the techniques developed for the study of avalanches in a previous work on the study of FLFO phase transitions in the random bond Ising model. ${ }^{9}$

The critical line can be found by studying two quantities: (i) the normalized average size $\left\langle\Delta m_{\max }\right\rangle$ of the largest avalanche in a cycle and (ii) the average duration $\left\langle t_{\max }\right\rangle$ of the largest avalanche in a cycle. As an example we show the behavior of these two quantities along a line with $p=0$ and changing the concentration $x$ (Fig. 11) and along a line with $x=0.375$ and changing the disorder parameter $p$ (Fig. 12). The phase transition is smoothed due to finite size effects,

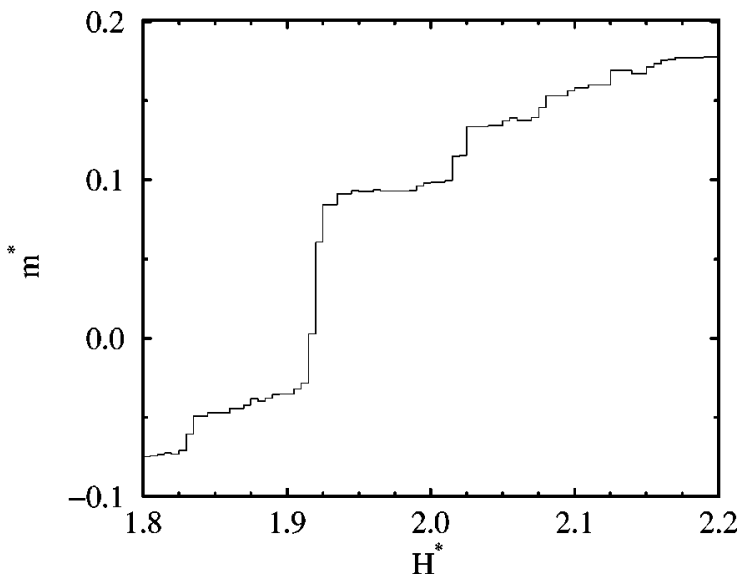

FIG. 9. Detail of the avalanches for a simulation with $x=0.25$ and $p=0.10$. Notice the existence of inverse avalanches, e.g., around $H^{*}=2.15$.

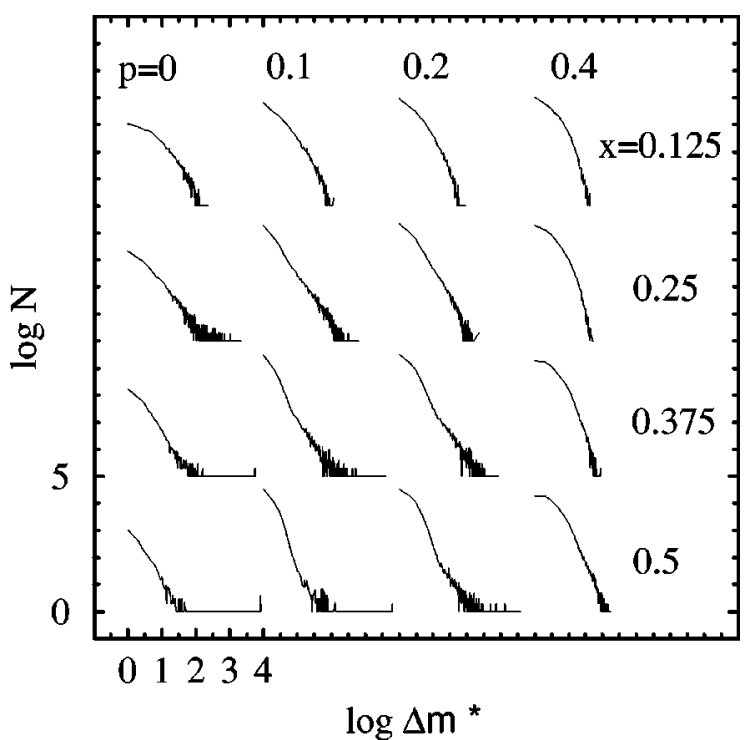

FIG. 10. Avalanche size distributions for different values of $p$ and $x$ as indicated.

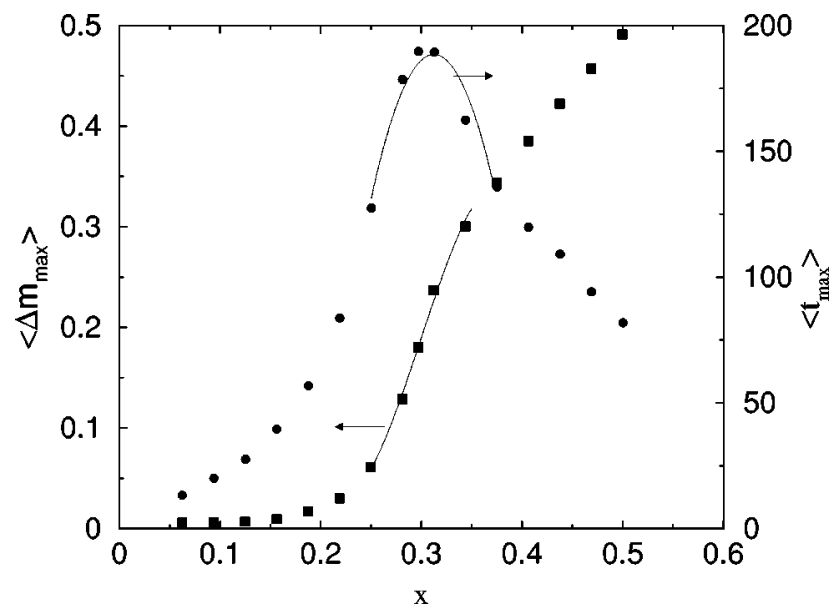

FIG. 11. Behavior of $\left\langle\Delta m_{\max }\right\rangle$ and $\left\langle t_{\max }\right\rangle$ as a function of $x$ for $p=0.0$.

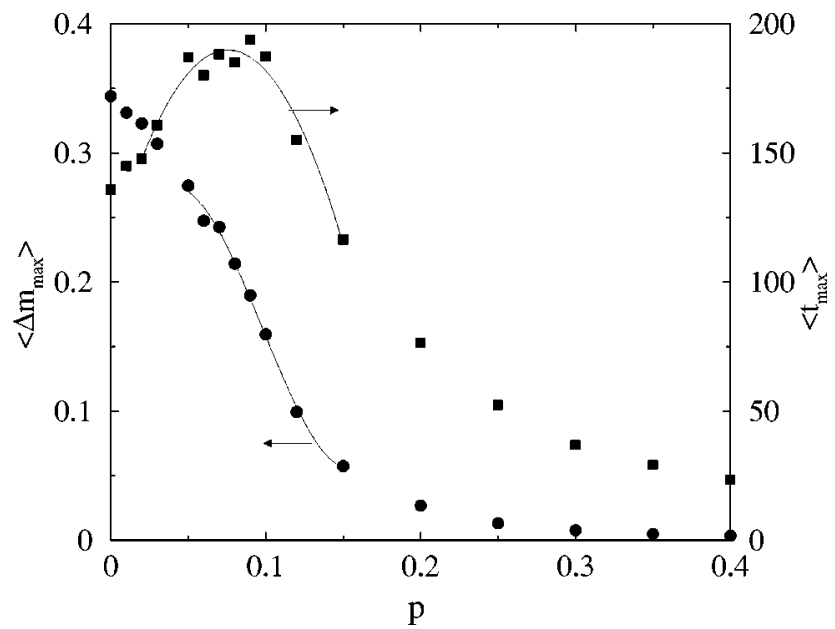

FIG. 12. Behavior of $\left\langle\Delta m_{\max }\right\rangle$ and $\left\langle t_{\max }\right\rangle$ as a function of $p$ for $x=0.375$. 


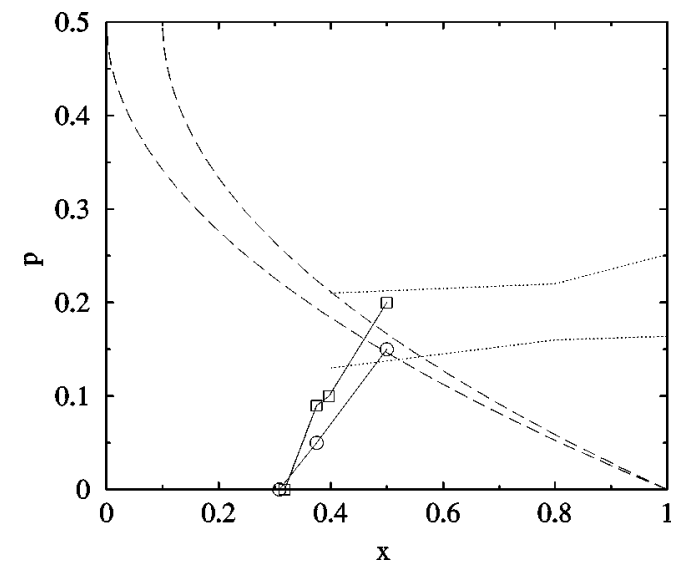

FIG. 13. $p-x$ diagram showing two different estimations of the position of the FLFO phase transition. Open squares correspond to the positions of the maximum in $\left\langle t_{\max }\right\rangle$ and open circles correspond to the position of the inflection points in $\left\langle\Delta m_{\max }\right\rangle$. Dotted lines correspond to two estimations of the $p(x)$ relation from MC simulations, as explained in the text. Long dashed lines correspond to estimations based on the behavior observed for the saturation magnetization

which can be corrected as explained in Ref. 9. Actually, estimations of the transition points can be obtained by locating, using a polynomial fitting, the maximum on $\left\langle t_{\max }\right\rangle$ and the inflection point on $\left\langle\Delta m_{\max }\right\rangle$. A systematic study of the behavior of these two quantities as a function of $x$ and $p$ allows us to obtain the phase diagram shown in Fig. 13. The discrepancy between the two estimations of the critical line can be attributed to finite-size effects. We find critical behavior, depending on $p$, for $x \geqslant 0.3$.

It is difficult to locate on this $p-x$ diagram the position where this phase transition will be expected to occur in the $\mathrm{Cu}-\mathrm{Al}-\mathrm{Mn}$ system, since the value of $p$ for our samples is unknown. Actually we can only have some rough estimations of the $p(x)$ dependence in the samples studied. First, it is possible to obtain $p$ from Monte Carlo simulations of the atomic ordering process in a realistic lattice model for $\mathrm{Cu}-$ Al-Mn presented in Ref. 16. The model considered in that paper contains only interactions to first and second nearest neighbors and its parameters have been fitted from the orderdisorder transition temperatures in the real experimental system. The behavior of $p$ as a function of $x$ obtained from that model is represented by the two dotted lines in Fig. 13. The lower line corresponds to the values of $p$ at $T=0$, and the upper represents the values of $p$ at temperatures just below the order-disorder transition. According to this result, the actual values of $p$ would be somewhere in the middle of these two dotted lines. The intersection of these lines with the phase transition (open symbols) would give the critical concentration where the change in the hysteresis cycle is expected. However, these estimations of $p(x)$ correspond to equilibrium configurations, but kinetic effects (samples have been quenched from high $T$ ) could modify the amount of disorder substantially. Moreover, the model used for the MC simulations does not contain any magnetic interaction term, which could have some relevance in the Mn ordering process. A second estimation can be obtained from the linear behavior of the saturation magnetization per atom $M_{s}$ with $x$ shown in Fig. 3. Note that the system contains two kinds of

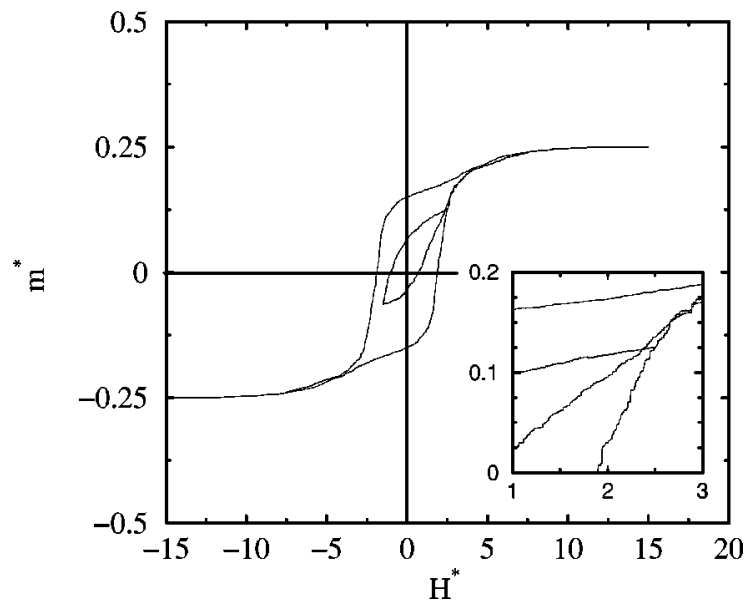

FIG. 14. Internal loops showing the absence of RPM property. Data correspond to a simulation with $x=0.25$ and $p=0.20$. The inset shows the detail of the turning point.

Mn atoms: the minoritary misplaced ones (in sublattice $\beta$ ) that exhibit antiferromagnetic interaction with its nearest neighbors and the majoritary ones (in sublattice $\alpha$ ) with ferromagnetic interaction with its second neighbors. Therefore, when reaching the remanence point by decreasing the applied field from saturation, the misplaced $\mathrm{Mn}$ atoms will present inverted magnetic moment. Thus, a naive approximation is $M_{s}=\mu\left[(1-B)(2 p-1)^{2}+B\right]$, where $\mu$ is the magnetic moment per atom and $\mu B$ is the saturation magnetization per atom corresponding to a sample with $\mathrm{Mn}$ atoms completely disordered over the $\alpha$ and $\beta$ sublattices ( $p$ $=0.5)$. Note that $M_{s}(p)$ is symmetric arround $p=1 / 2$ as can be expected. The above equation combined with the linear behavior $M_{s} \simeq \mu x$ presented in Fig. 3, leads to a parabolic dependence $x=\left[(1-B)(2 p-1)^{2}+B\right] .^{30}$ Although the value of $B$ is unknown, it should be very small. At least smaller than the value $M_{s}=0.1 \mu$ found for our sample with $x=0.12$. We can, therefore, sketch this behavior on the $p-x$ diagram shown in Fig. 13. The intersection of the two parabolas (corresponding to $B=0.1$ and $B=0$ ) with the transition line found from our model gives a different estimation of the transition point.

Taking into account the above arguments, from Fig. 13 it seems reasonable to assume that the transition is located at $x_{c} \sim 0.5 \pm 0.1$. Although this value is slightly above the experimental estimation $x_{c} \sim 0.3$, the values are still close enough to suggest that, given the similarity of the effects observed in the experimental and simulated hysteresis cycles, the phenomenon may be an example of the kind of phase transition found in the model.

From the model developed in the present work, we can also study the internal hysteresis loops in order to compare with the behavior found experimentally. Figure 14 shows, as an example, an internal loop for a system with $x=0.25$ and $p=0.2$. The qualitative agreement with the experimental case is extremely good, and in both cases an increase of the magnetization is found after the internal loop. This shows that the RPM property does not hold. Actually, two conditions must be satisfied for the RPM property to be fulfilled. The first one is the adiabatic character of the system evolution and the second one is the so-called no-passing rule (preservation of partial ordering of metastable states by the 
dynamics). ${ }^{31}$ The former condition is satisfied in our simulations (synchronous dynamics) and approximately in the experiments (slow driving rate). Nevertheless, the existence of antiferromagnetic interactions producing inverse avalanches (see Fig. 9) destroys the second condition.

\section{B. Polycrystallinity}

The model developed above describes the behavior of a single crystal with an easy axis of magnetization (EAM) that corresponds to the direction of the external field. In a polycrystalline sample the situation is quite different: grains present different crystallographic orientations with respect to the applied field. The corresponding angular distribution of EAM results on two main effects on the hysteresis cycles: (i) the cycles become smoother (hence the avalanches are much more difficult to detect) and (ii) the hysteresis cycle changes its shape. We can estimate this change in the case of uniaxial anisotropy. Let $m_{0}^{*}\left(H^{*}\right)$ be the cycle corresponding to a single crystal oriented with the EAM along the external field $H^{*}$, and let $m^{*}\left(H^{*}\right)$ the cycle corresponding to the polycrystal. Assuming that there is a uniform angular distribution of EAM in the polycrystal, we will have

$$
m^{*}\left(H^{*}\right)=\int \cos \theta m_{0}^{*}\left(H^{*} \cos \theta\right) d \Omega,
$$

where $d \Omega$ is the differential solid angle. This integral has to be performed over all the possible directions of the EAM in the sample. This is not straightforward and requires the knowledge of the direction of the EAM. For a Cu-Al-Mn single crystal it is not known. Nevertheless we can obtain an approximate solution to Eq. (7) as

$$
m^{*}\left(H^{*}\right) \simeq \int_{0}^{\theta_{0}} \cos \theta m_{0}^{*}\left(H^{*} \cos \theta\right) 2 \pi \sin \theta d \theta,
$$

where the factor $2 \pi \sin \theta d \theta$ is the differential of solid angle and $\theta_{0}$ is the maximum angle between the field and the EAM. For instance, we have $\cos \theta_{0}=\sqrt{3} / 3$ for a (100) and (111) EAM, and $\cos \theta_{0}=\sqrt{2} / 2$ for a (110) EAM. In the extreme case of a monoclinic symmetry (a single EAM) one will have $\cos \theta_{0}=0$. Figure 15 shows the results of this correction on a given hysteresis cycle. As expected the jump at the phase transition becomes broadened and extends in a certain range of fields.

\section{DISCUSSION AND CONCLUSIONS}

In Sec. II we have presented low-temperature measurements of the hysteresis cycles of $\mathrm{Cu}-\mathrm{Al}-\mathrm{Mn}$ in three samples of different Mn content. We have shown that for temperature $T \lesssim 5 \mathrm{~K}$ and Mn content $x>0.3$ the loops exhibit a sharp reversal of magnetization in their central part. We have proposed that this change is related to the existence of a phase transition from a spin-glass phase to a ferromagnetic spinglass phase.

In order to gain insight into this phase transition we have proposed (in Sec. III) a model for the study of the magnetic properties of this system, including the basic physical ingredients of the problem. The model is based on a $T=0$ diluted Ising model with exchange interactions that have been fitted

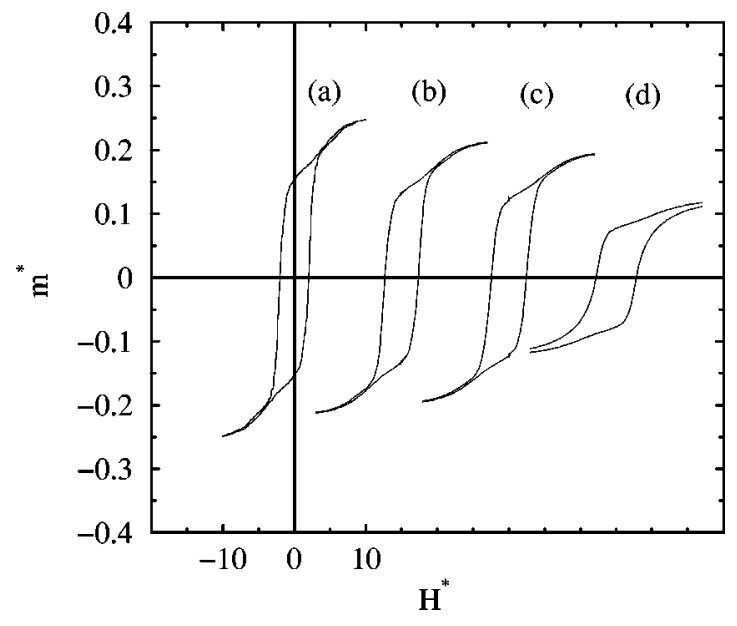

FIG. 15. Effect of the uniaxial anisotropy on the hysteresis cycle of polycrystalline systems. Data correspond to the same simulation as in Fig. 15. The different cycles correspond to: single crystal (a), $\cos \theta_{0}=\sqrt{2} / 2$ (b), $\cos \theta_{0}=\sqrt{3} / 3$ (c), and $\cos \theta_{0}=0$ (d).

to the experimental values corresponding to stoichiometric $\mathrm{Cu}_{2} \mathrm{AlMn}$. The model reproduces the existence of magnetic disorder arising from the nonstoichiometry and the misplaced Mn atoms, as found experimentally. It should be remarked that we have not studied the exact equilibrium ground state of the model. Rather we have focused on the hysteresis loops that are obtained by using a synchronous dynamics evolution that drives the system through a sequence of metastable states.

Although the shape of the loops has not been perfectly reproduced by the model, some of the relevant features of the real loops have been found on the simulated ones. This includes the following.

(1) The change from smooth to sharp cycles by changing the Mn content. In the simulations this change corresponds to a phase transition, of the same kind as the one reported in the random field ${ }^{11}$ and random bond Ising models, ${ }^{9}$ and it is found at $x=0.5 \pm 0.1$, which is close to the experimental estimation $x \sim 0.3$

(2) The existence of long tails for large fields. The experimental cycles are not saturated even at fields of $5 \mathrm{~T}$. From the model, we have deduced that these tails arise (i) from the existence of minoritary antiferromagnetic interactions and (ii) as a consequence of the polycrystalline character of the samples used in the experiments. The magnetic moments subjected to an effective antiferromagnetic interaction are very diluted and do not exhibit any cooperative behavior. Consequently they show a reversible response to the external field. From the model, it is reasonable to suppose that these magnetic moments are associated to misplaced $\mathrm{Mn}$ atoms having other $\mathrm{Mn}$ atoms as nearest neighbors. Concerning the polycrystallinity of the samples, its main effect is the broadening of the hysteresis due to the averaging over all possible crystallographic orientations of the grains with respect to the external field.

(3) The fact that the RPM effect is not satisfied has been found both experimentally and in the simulations. The reason is the existence of inverse avalanches associated with the presence of antiferromagnetic interactions. In these avalanches $d M / d H$ is negative, contrary to the stability condi- 
tions deduced from equilibrium thermodynamics. The possibility of such a result is due to the fact that the process considered is not an equilibrium one (even if it is very close to a quasistatic process). In fact the system evolves through a series of metastable states separated by large energy barriers. In the simulations metastability arises from the fact that relaxation is performed according to the local effective field acting on each magnetic moment.

An important question to be answered is why avalanches are not observed in the experiments. It is clear that polycrystallinity limits the maximum size of the avalanches to that of a grain. Given that the typical size of the grains is of the order of $100 \mu \mathrm{m}$, and the volume of the sample is $\sim 3 \mathrm{~mm}^{3}$, one will need a relative resolution much better than 3 $\times 10^{-4}$ in the magnetization measurement. This means a resolution better than $0.1 \mathrm{emu} /(\mathrm{mol} \mathrm{Mn})$, while the actual resolution of our experimental system is of the order of 10 emu/(mol Mn). The inset of Fig. 7 is illustrative of this point. It would be very enlightening to perform experiments in single crystals, where we expect that avalanches could be observed.

Concerning the phase transition found in the model it is interesting to discuss several points. First, as mentioned before, the phase transition found in our diluted Ising model (and in other similar diluted Isind models ${ }^{25}$ ) is of the same kind as those found for the random field ${ }^{11}$ and random bond Ising models. ${ }^{9}$ For these last two models it is known that the phase transition in the hysteresis loop is associated with an equilibrium ground state phase transition. ${ }^{32}$ It is reasonable to think that for our version of the site diluted Ising model, there will also exist such a ground state transition from a completely disordered ground state (small $x$ region) to a ground state with a nonvanishing magnetization (large $x$ region). Experimentally, there are evidences that in $\mathrm{Cu}-\mathrm{Al}-\mathrm{Mn}$ these two phases correspond to a true spin-glass phase and to a ferromagnetic spin glass. ${ }^{19}$ In the model, whether the two phases can be catalogued as being spin-glass-like would depend on its thermal behavior. This study is out of the scope of the present work.

Secondly, the transition line in the $p-x$ diagram has been located from the maximum of the duration and the inflection of the size of the largest avalanche. An additional estimation can be obtained by locating the points where the distribution of avalanche sizes becomes power law. Except for finite-size effects, the three estimations should be coincident. Obtaining the transition points accurately using the third method is quite difficult because it is necessary to perform nonlinear fits of a power-law with an exponential correction, $N(s)$ $\sim s^{-\tau} e^{\lambda s}$, in order to locate the point $(\lambda=0)$ where the distribution changes from supercritical $(\lambda>0)$ to subcritical $(\lambda<0)$ behavior. It is much easier to evaluate the exponent $\tau$ characterizing the critical distribution of avalanches, which is not affected by the small exponential corrections close to the critical line. The obtained value is $\tau=1.7 \pm 0.1$. This value can be compared with the values $\tau=1.8 \pm 0.2$ and 2.0 \pm 0.2 found for the three-dimensional (3D) random field Ising model and the 3D random bond Ising model. ${ }^{10}$ This agreement of the numerical values reinforces the hypothesis that there exists universality in these kind of models exhibiting FLFO phase transitions.

Finally, a much deeper question concerns the nature of this critical line. Some authors have stated that critical distributions of avalanches do not occur at a certain line but on a broad region due to the existence of the so-called selforganized criticality. This theory has been proposed for externally driven complex dissipative systems with spatial and temporal degrees of freedom. According to Bak et al. ${ }^{33}$ these systems naturally evolve to a critical state characterized by avalanches with no intrinsic time and length scales. This results in power-law distributions for avalanches. In this sense the results we have obtained from the model point in the opposite direction: we are dealing with a true critical line. Indeed, for selected values of the model parameters controlling the disorder $p$ and $x$, the system exhibits the critical behavior, as can be seen in Fig. 10.

\section{ACKNOWLEDGMENTS}

We acknowledge Benjamín Martínez for a helpful collaboration and a critical reading of the manuscript. We also thank Ll. Mañosa, J. Ortín, and C. Frontera for fruitful discussions. This work has received financial support from CICyT (Spain) Project No. MAT98-0315, and CIRIT (Catalonia) Project No. SGR119.
${ }^{1}$ G. Bertotti, Hysteresis in Magnetism (Academic, New York, 1998).

${ }^{2}$ A. H. Barkhausen, Z. Phys. 20, 401 (1919).

${ }^{3}$ R. Vergne, J. C. Cotillard, and J. L. Porteseil, Rev. Phys. Appl. 16, 449 (1981).

${ }^{4}$ P. J. Cote and L. V. Meisel, Phys. Rev. Lett. 67, 1334 (1991); J. S. Urbach, R. C. Madison, and J. T. Markert, ibid. 75, 276 (1995).

${ }^{5}$ E. Vives, J. Ortín, L1. Mañosa, I. Rafols, R. Pérez -Magrané, and A. Planes, Phys. Rev. Lett. 72, 1694 (1994)

${ }^{6}$ W. Wu and P. W. Adams, Phys. Rev. Lett. 74, 610 (1995).

${ }^{7}$ M. P. Lilly, A. H. Wootters, and R. B. Hallock, Phys. Rev. Lett. 77, 4222 (1996).

${ }^{8}$ G. Bertotti and M. Pasquale, J. Appl. Phys. 67, 5255 (1990).

${ }^{9}$ E. Vives and A. Planes, Phys. Rev. B 50, 3839 (1994).
${ }^{10}$ E. Vives, J. Goicoechea, J. Ortín, and A. Planes, Phys. Rev. E 52, R5 (1995).

${ }^{11}$ J. P. Sethna, K. Dahmen, S. Kartha, J. A. Krumhansl, B. W. Roberts, and J. D. Shore, Phys. Rev. Lett. 70, 3347 (1993).

${ }^{12}$ K. L. Babcock and R. M. Westervelt, Phys. Rev. Lett. 64, 2168 (1990).

${ }^{13}$ L1. Carrillo, L1. Mañosa, J. Ortín, A. Planes, and E. Vives, Phys. Rev. Lett. 81, 1889 (1998).

${ }^{14}$ J. Kushauer, R. van Bentum, W. Kleemann, and D. Bertrand, Phys. Rev. B 53, 11647 (1996).

${ }^{15}$ P. Cizeau, S. Zapperi, G. Durin, and H. E. Stanley, Phys. Rev. Lett. 79, 4669 (1997).

${ }^{16}$ E. Obradó, C. Frontera, Ll. Mañosa, and A. Planes, Phys. Rev. B 58, 14245 (1998).

${ }^{17}$ P. J. Webster and K. R. A. Ziebeck, in Heusler Alloys, Landolt- 
Bornstein New Series, Vol. III/19c, edited by O. Madelung (Springer-Verlag, Berlin, 1988), p. 75.

${ }^{18}$ K. Tajima, Y. Ishikawa, P. J. Webster, M. W. Stringfellow, D. Tochetti, and K. R. A. Ziebeck, J. Phys. Soc. Jpn. 43, 483 (1977).

${ }^{19}$ E. Obradó, A. Planes, and B. Martínez, Phys. Rev. B 59, 11450 (1999).

${ }^{20}$ N. Nakanishi, T. Shigematsu, M. Machida, K. Ueda, K. Shimitzu, and Y. Nakata, in Proceedings of the International Confernece on Martensitic Transformations '92, edited by C. M. Wayman and J. Perkins (Monterey Institute for Advanced Studies, Monterey, CA, 1993), p. 581.

${ }^{21}$ M. O. Prado and F. C. Lovey, Acta Mater. 46, 137 (1998).

${ }^{22}$ J. J. Préjean, M. J. Joliderc, and P. Monod, J. Phys. (Paris) 41, 427 (1980).

${ }^{23}$ By "ferromagnetic spin-glass", we understand a phase which, in absence of an applied field, exhibits a large degree of frozen disorder but with clusters with nonzero magnetization. Such structures have also been called cluster glasses or mictomagnetic phases. See, for instance, C. M. Hurd, Contemp. Math. 23, 469 (1982).

${ }^{24}$ K.H. Fischer and J.A. Hertz, Spin Glasses (Cambrige University Press, Cambridge, 1991).
${ }^{25}$ B. Tadić, Phys. Rev. Lett. 77, 3843 (1996).

${ }^{26}$ Actually the structure of the system in the martensitic phase is monoclinic but, due to the diffusionless character of the martensitic transition, the atomic environment is very similar in both the bcc and martensitic phase.

${ }^{27}$ Such a value can be obtained by assuming a number of conduction electrons per Mn atom $n_{c}=8$.

${ }^{28}$ P. Gibbs, T. M. Harders, and J. H. Smith, J. Phys. F 15, 213 (1985).

${ }^{29}$ Since there are antiferromagnetic interactions, inverse avalanches may occur and, in few occasions, a degenerate situation may appear with two energetically equivalent configurations which successively alternate. In such cases we decide randomly which is the stable one and proceed with the field increment.

${ }^{30}$ It is clear from this expression that the approximation is not valid for very low values of $x$.

${ }^{31}$ A. A. Middleton, Phys. Rev. Lett. 68, 670 (1992).

${ }^{32}$ A. T. Ogielski, Phys. Rev. Lett. 57, 1251 (1986); M. R. Swift, A. J. Bray, A. Maritan, M. Cieplak, and J. R. Banavar, Europhys. Lett. 38, 273 (1997).

${ }^{33}$ P. Bak, C. Tang, and K. Wiesenfeld, Phys. Rev. Lett. 59, 381 (1987); Phys. Rev. A 38, 364 (1988). 